\title{
LITHIUM MICRODIALYSIS AND ITS USE FOR MONITORING OF STOMACH AND COLON SUBMUCOSAL BLOOD PERFUSION - A PILOT STUDY USING ISCHEMIC PRECONDITIONING IN RATS
}

\author{
Norbert Cibiček ${ }^{1}$, Stanislav Mičuda ${ }^{2}$,Jaroslav Chládek ${ }^{2}$, Pavel Živný1, Zdeněk Zadák ${ }^{3}$, Eva Črmáková4, Vladimír Palička ${ }^{1}$ \\ Charles University in Prague, Faculty of Medicine in Hradec Králové and University Hospital Hradec Králové, Czech \\ Republic: Institute for Clinical Biochemistry and Diagnostics ${ }^{1}$; Department of Pharmacology²; Department of Gerontology \\ and Metabolism, Research Laboratory ${ }^{3}$; Department of Biophysics and Biostatistics ${ }^{4}$
}

\begin{abstract}
Summary: During shock, exposure of gut to ischemia determines patient's survival. Ischemic preconditioning (ISP) elevates nitric oxide and blood perfusion, whereby it protects organs against subsequent severe ischemia/reperfusion. Using appropriate flow marker, microdialysis may serve to monitor interstitial microcirculation. Hence, our aim was to test the reliability of lithium as a flow marker (lithium microdialysis, LM) on an ISP model. Rats were divided into three groups. Two (ischemic and preconditioned) groups underwent 30 min celiac artery occlusion (CAO) with $2.5 \mathrm{~h}$ reperfusion. 25 min before $\mathrm{CAO}$, the latter experienced $5 \mathrm{~min}$ ischemia. Sham-operated animals served as controls. LM in stomach and colon submucosa, serum nitric oxide, hepatic and pancreatic enzymes were measured. In stomach, LM indicated a decrease in blood perfusion evoked by CAO $(\mathrm{p}<0.01)$ in both experimental groups. During reperfusion, the ischemic animals showed a restoration of microcirculation, unlike the preconditioned ones, whose blood perfusion failed to regenerate $(\mathrm{p}<0.001)$. For any group, LM showed no microcirculation modification in colon. Serum analytes remained unchanged. We conclude that LM appears to be a potentially suitable indicator of gastrointestinal interstitial microcirculation. However, we failed to demonstrate any beneficial effect of ISP on pancreas, systemic nitric oxide and local/remote microcirculation within studied organs.
\end{abstract}

Key words: Microdialysis; Microcirculation; Gastrointestinal tract; Ischemic preconditioning

\section{Introduction}

It is well recognized that in critical situations, the prognosis of patients depends largely on maintenance of adequate blood perfusion in order to preserve the barrier integrity and metabolic functions of gastrointestinal tract and its adjacent organs. Recent evidence has shown that the viability of gut exposed to ischemic insult can be enhanced by ischemic preconditioning (ISP) taking place prior to sustained ischemia $(1,3)$. Because of deleterious impact ischemia in splanchnic organs may exert on the entire organism via bacterialand toxin translocation and cytokine production, ISP offers an interesting therapeutic approach for clinical practice (14).

ISP refers to a process by which a (series of) brief ischemic episode(s) confers protection against subsequent prolonged ischemia/reperfusion. Regarding the mechanism, a number of neurohumoral mediator pathways have been proposed, where elevated nitric oxide followed by enhanced blood perfusion plays a central role (14). ISP can be examined with respect to one specific organ, or considering other organs at a distance. The former differentiates between local and regional ISP, whereas the latter defines an inter-organ or remote ISP (rISP). With respect to abdominal organs, ISP has been exemplified in the liver (14), pancreas (3), small intestine (10) or stomach (1). However, rISP in colon has not attracted much interest, yet.

In many organs including the gut, in vivo microdialysis is a well-established miniinvasive means of local tissue metabolism monitoring (2). Using appropriate flow marker (also called tracer), this method offers opportunity to assess tissue microcirculation based on an assumption that the washout of tracer from the probe is related to extracellular fluid kinetics, which depends primarily on microcirculatory conditions. However, the most frequent tracer molecule (ethanol) is volatile, its precise analytical determination may require sophisticated instruments (like mass spectrometer), or needs to be labeled radioactively (15). Recently, a novel, apyrogenic, non-radioactive and freely diffusible flow marker with well-defined toxicity in humans - lithium, has successfully been applied to describe blood perfusion modifications in rat liver, kidney and muscle 
interstitium (7). Nevertheless, further information on the usefulness of this promising tracer are needed.

Hence, the aim of this study was twofold: first, to verify the hypothesis that lithium could be used for monitoring of microcirculation in the alimentary tube and second, to evaluate the influence of celiac ISP on celiac artery occlusion (CAO)-induced microcirculatory changes in stomach and descending colon. Both hypotheses were tested on a rat model of gastric ischemia and ISP performed at the level of celiac artery trunk (1). Furthermore, the systemic effects of ISP and CAO were assessed using selected serum enzyme activities and nitric oxide concentration.

\section{Material and Methods}

Materials. Flame photometer serum-standard solution (Eppendorf, Hamburg, Germany) with $\mathrm{Li}^{+}$concentration of $2.00 \mathrm{mmol} \mathrm{l}^{-1}$ was used as microdialysis perfusion medium.

Animals. Adult male Wistar rats weighing 260 to $330 \mathrm{~g}$, were used. The animals were housed in the animal quarters under controlled environmental conditions. They had free access to standard rat chow except 16-18 hours before experiments, when they were fasted. Tap water was provided ad libitum. The rats received care in accordance with the guidelines set by the Institutional Animal Use and Care Committee of the Charles University in Prague, Czech Republic. All experimental procedures were approved by local ethics committee.

Experimental protocol. The animals were randomly assigned to three groups denoted as S, IS, and ISP ( $\mathrm{n}=6-10$ in each group). The first group (S) was sham-operated. The second group (IS) underwent a 30 min period of complete CAO with subsequent $2.5 \mathrm{~h}$ of reperfusion. The rats in the third group (ISP) were preconditioned by exposure to a short ischemia/reperfusion period ( 5 and $25 \mathrm{~min}$, respectively), followed by prolonged ischemia/reperfusion (30 min and $2.5 \mathrm{~h}$, respectively). During all procedures, the animals were under general i.p. pentobarbital anesthesia (50 mg kg-1 followed by $15 \mathrm{mg} \mathrm{kg}^{-1} \mathrm{~h}^{-1}$ ) and operated in supine position. The rectal temperature was monitored and maintained at $38.5^{\circ} \mathrm{C}$ with a heating lamp. The trachea was cannulated to ensure patent airways. After midline laparotomy, the celiac artery was disclosed and underlaid by smooth rubber tubing to assist later clamping. Ischemia/reperfusion was accomplished by placement/removal of a microbulldog clamp at the level of celiac artery origination from abdominal aorta. The success of each intervention was verified visually (assessment of blood flow distal from the site of occlusion).

Gastric and colon submucosal microdialysis technique. After laparotomy, stomach was exteriorized and moistened continuously. Avoiding blood vessels, a $6 \mathrm{~mm}$ long tunnel was made from serosal aspect in the submucosal layer of its glandular part from greater to lesser curvature by means of a $26 \mathrm{G}$ needle. For the descending colon, similar procedure was followed along with its long axis at a distance of $5 \mathrm{~cm}$ from the anus. Into the tunnels, microdialysis probes (CMA/20, active length $4 \mathrm{~mm}$, outer diameter $0.5 \mathrm{~mm}$, cutoff $20 \mathrm{kDa}, \mathrm{CMA} /$ Microdialysis, Solna, Sweden) were carefully inserted and fixed in place with sutures. After each experiment, probes were tested for leakage and their former positions were verified by histology (Fig. 1). After surgery, the abdominal opening was closed to avoid fluid losses. Microdialysis catheters were perfused at $1.2 \mu \mathrm{min}^{-1}$ using a perfusion pump. An initial $60 \mathrm{~min}$ stabilisation period was followed by $5 \mathrm{~h}$ of experimental procedure (see above) with continuous dialysate sampling in $30 \mathrm{~min}$ intervals into microvials. Samples were stored at $-20{ }^{\circ} \mathrm{C}$ until lithium determination. At the end of experiment, arterial blood was withdrawn and the removed serum was stored at $-20^{\circ} \mathrm{C}$ until analysis.

Blood perfusion measurement. The extent of submucosal blood perfusion was expressed as lithium inflow - outflow concentration $\left(\mathrm{Li}_{\text {in-out }}\right)$ difference, i.e. Li efflux as reported previously (7). Dialysate $\mathrm{Li}^{+}$was determined using EFOX 5053 flame photometer (Eppendorf, Hamburg, Germany) according to manufacturer's instructions.

Determination of nitric oxide in serum. Nitric oxide was measured indirectly (and expressed) as nitrate and nitrite (the stable nitric oxide metabolites) using HPLC with UV-VIS detection and fluorimetry methods described elsewhere (8).

Determination of serum enzyme activities. Hitachi 917 autoanalyser (Boehringer, Mannheim, Germany) with commercially available reagent kits (Roche Diagnostics GmbH, Mannheim, Germany) were utilized. For the study of the extent of liver injury, alaninaminotransferase (ALT), aspartataminotransferase (AST), lactatdehydrogenase (LDH) and cholinesterase (CHE) serum activities were investigated. To evaluate the level of pancreatic damage, lipase (LIP) and amylase (AMYL) activities were determined. All analyses were performed in accordance with manufacturer's instructions.

Histology. The removed tissues were fixed in $10 \%$ formaline, embedded in paraffin and further treated according to procedures standard for hematoxylin-eosine stain.

Statistics. Data are expressed as means \pm standard error of mean (SEM). For statistical evaluation, Analysis Of Variance with Fisher's LSD multiple comparison post hoc test (programs NCSS 2004 and Statistica) were used. The chosen level of significance was $\alpha=0.05$.

\section{Results}

Blood perfusion estimation. Fig. 2 summarizes the time course of $\mathrm{Li}_{\text {in-out }}$ differences in all groups in the submucosa of stomach (a) and colon (b). The zero interval of microdialysate collection (obtained within 60-90 min after commencement) provided basal values, where no 
statistically significant difference was found between the stomach and colon. Likewise, between-group comparisons showed comparable baseline values in stomach as well as in colon.

Blood perfusion in stomach (Fig. 2a). In the S group, absolute values of $\mathrm{Li}_{\text {in-out }}$ difference showed no variation in time. Brief ( $5 \mathrm{~min}$ ) ischemia in the ISP group caused a significant drop in $\mathrm{Li}_{\text {in-out }}$ difference from its baseline $(p<0.05)$. Within the IS and ISP groups, 30 min interval of $\mathrm{CAO}$ produced significant depressions in $\mathrm{Li}_{\text {in-out }}$ difference when compared with corresponding basal levels $(p<0.01)$. Immediately after onset of reperfusion, the values of $\mathrm{Li}_{\text {in-out }}$ difference raised in both groups, reaching levels not significantly different from their baselines. In the next 2 hours, $\mathrm{Li}_{\text {in-out }}$ difference within the IS group continued to elevate slowly, while the opposite holds true for ISP group, where we observed a steadily falling trend $(p<0.001)$. However, none of the changes observed within the ligated groups was

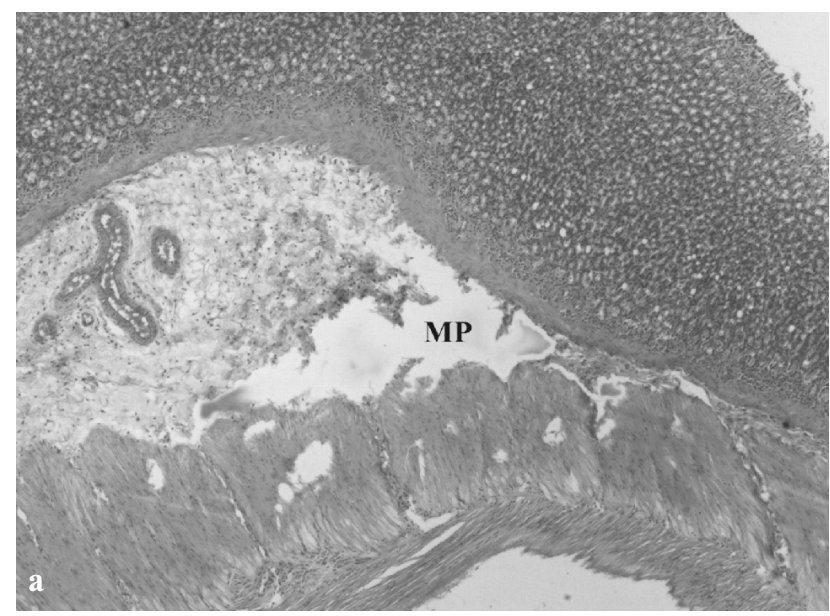

sufficient to statistically manifest also in between-group comparisons with controls.

Blood perfusion in descending colon (Fig. 2b). Similar to gastric perfusion, the $\mathrm{S}$ group provided constant values of $\mathrm{Li}_{\text {in-out }}$ difference over the entire experiment. Neither within-group, nor between-group comparisons in parallel time intervals disclosed differences reaching the level of significance.

Serum nitric oxide and enzymatic activities. The extent of potential organ impairment and modulation of the body's metabolism was evaluated by determination of selected serum analytes. Fig. 3 depicts the grade of nitric oxide production modulation by ischemia and ISP. Even though the mean serum concentration of nitric oxide in IS group was $165 \%$ of that in control group (S), the difference was only marginally significant $(\mathrm{p}=0.06)$. The ISP group gave results similar to controls. Likewise, the activities of serum enzymes in question were not statistically different (Fig. 4).

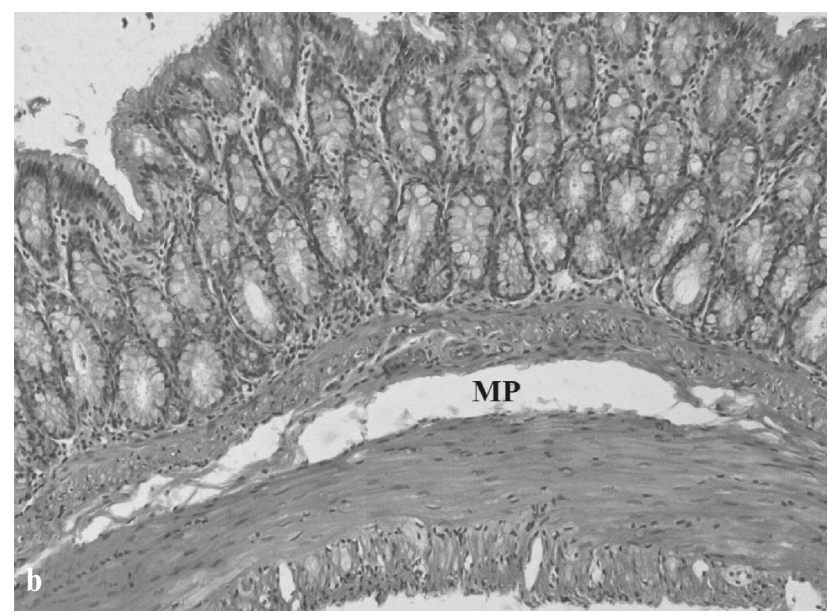

Fig. 1: Histological appearance of the wall of stomach (panel a) and descending colon (panel b) - a tangential section to previous probe positions marked MP ("microdialysis probe"). Sham-operated group, haematoxylin-eosine stain.
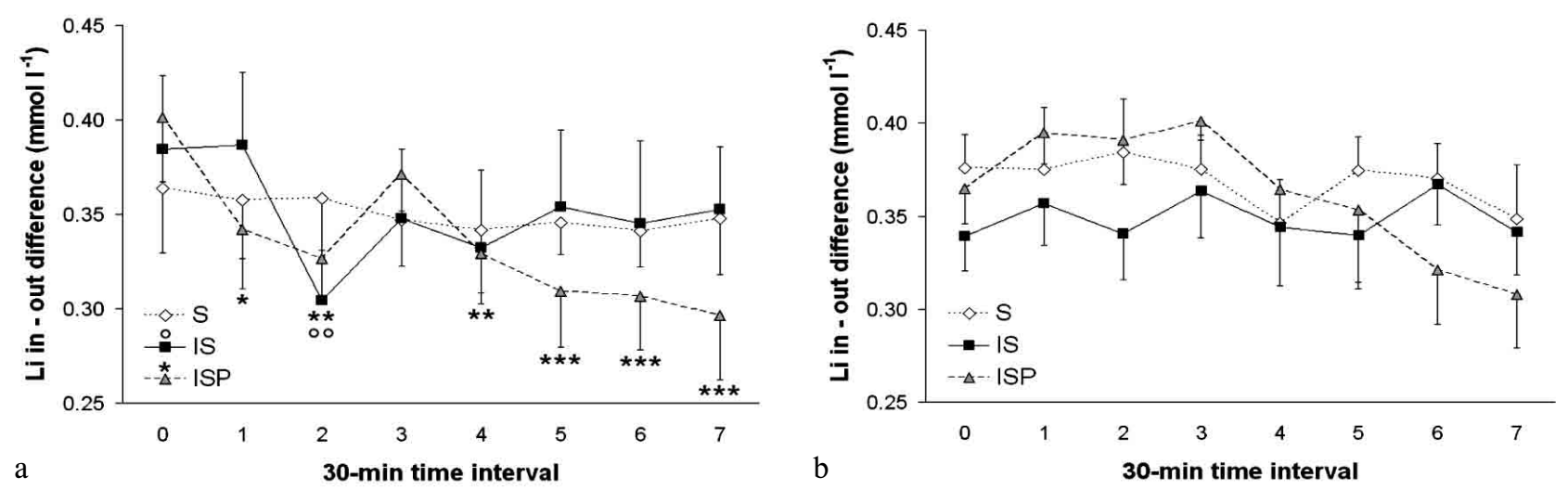

Fig. 2: A comparison of submucosal stomach (a) and colon (b) blood perfusion among the three groups (S, IS, ISP) followed in time and expressed as $\mathrm{Li}_{\text {in-out }}$ difference. Within the groups, markings ${ }^{*},{ }^{\circ} ;{ }^{* *},{ }^{\circ}$ and $* * *,{ }^{* 00}$ denote $\mathrm{p}<0.05 ; \mathrm{p}<0.01$ and $p<0.001$, respectively as compared to corresponding reference (baseline) levels. Results are mean \pm SEM of 6-8 rats ( 2 animals excluded due to hemorrhage around the probe). 


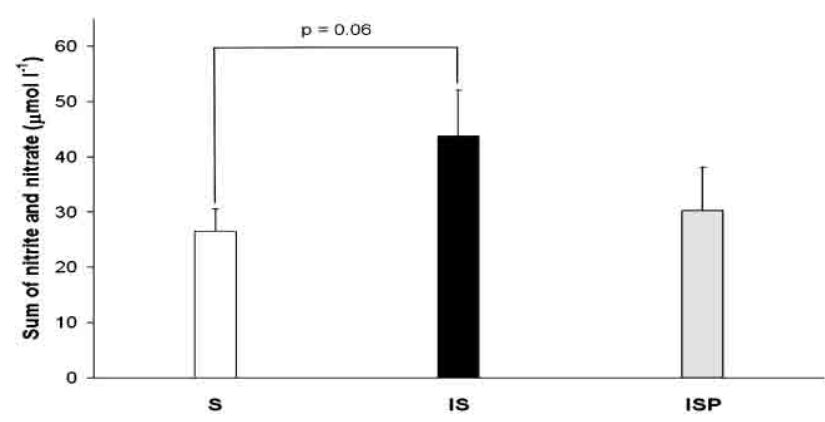

Fig. 3: Effect of ischemia (IS) and ischemic preconditioning (ISP) on serum nitric oxide (shown as the sum of nitrite and nitrate and compared with sham-operated group, $\mathrm{S}$ ). Results are mean + SEM of 6-10 rats.

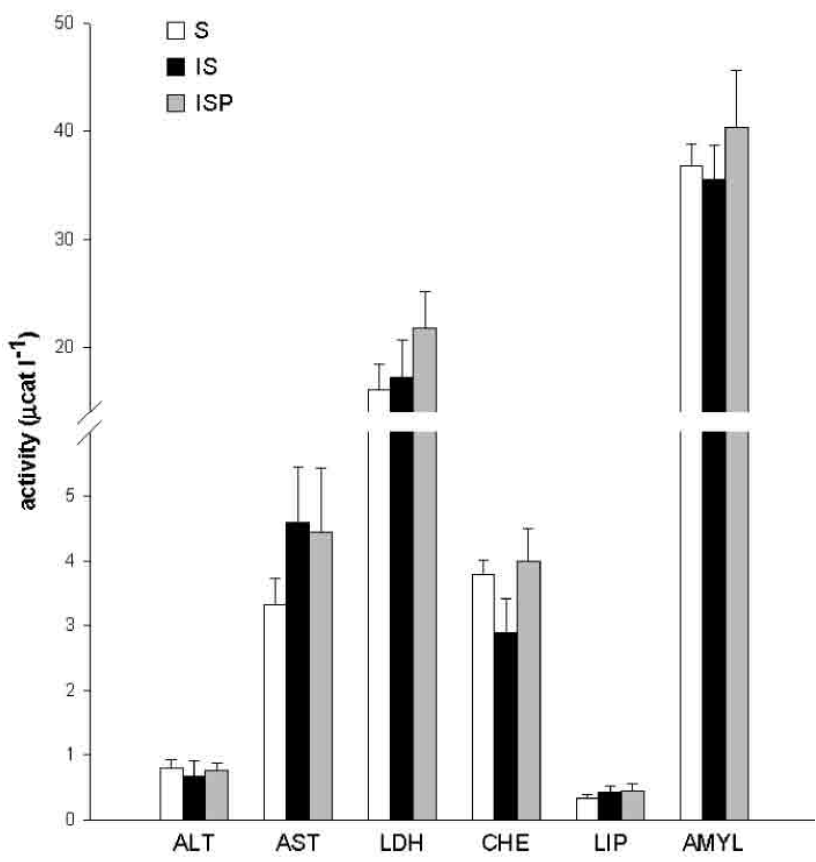

Fig. 4: Effects of ischemia (IS) and ischemic preconditioning (ISP) on serum enzyme activities (expressed as comparisons with sham-operated group, S). Results are mean + SEM of 6-10 rats. Level of significance was not reached in any of the studied parameters.

\section{Discussion}

This pilot work presents a new modification of blood perfusion measurement using microdialysis in two distinct parts of rat alimentary tube. Our most important findings were twofold: first, the anticipated stability of $\mathrm{Li}_{\text {in-out }}$ difference in the $\mathrm{S}$ group and its response to ischemic periods in the experimental groups and second, the unexpected decrease in $\mathrm{Li}_{\text {in-out }}$ difference observed during reperfusion in the ISP group as well as statistically invariable serum levels of nitric oxide and enzyme activities.
In sham-operated animals, the stability of $\mathrm{Li}_{\text {in-out }}$ difference indicates a steady state of microcirculation throughout the experiment. The decreases seen in stomach during ischemia and/or ISP in respective groups correspond to the changes induced by CAO. In the course of reperfusion, the flow marker was indicating either a predictable tendency of stomach microcirculation to return to the initial pre-ischemic levels (IS group), or after a short-term rise displayed an unexpected deterioration of tissue microcirculation till the end of experiment (ISP group). This is contrary to studies which, using other techniques, recognized preservation of postischemic microcirculation as one of the main mechanisms of protection elicited by ISP $(1,10)$. In addition, applying this method, there was no detectable change in tissue perfusion of descending colon suggesting practically no effect of remote (stomach) ischemia and/or ISP in this part of alimentary tract. Such finding is interesting since beneficial relations due to ISP were found to exist in many distant organs with virtually no difference between local and remote effects as to potency $(4,1)$.

In general terms, the mechanism of local/remote effects of ISP is likely to be dependent on signalling pathways including systemic elevation of nitric oxide production, whereas pure sustained ischemia is associated with a decline in nitric oxide level (9). We did not observe the proclaimed hemodynamic effects of ISP, but concurrently neither did we detect a significant rise in serum nitric oxide metabolites in the ISP group. This might to some extent be explained by the adenosine/xanthine theory, which would suggest an inadequate reperfusion phase during preconditioning in the third group. Be that the case, instead of blood perfusion improvement, ISP would have resulted in longer and more severe $(1 \mathrm{~h})$ ischemia with accumulation of disproportional amounts of xanthine leading to production of excessive superoxide anion able to remove the generated nitric oxide (13).

With regard to the time window for ISP, it was documented, that one 5 min episode of arterial occlusion effectuated $30 \mathrm{~min}$ in advance of prolonged ischemia was sufficient to reduce lesion area in rat stomach (12). In small bowel, Hotter et al. (6) produced comparable effects (nitric oxide generation, organ protection) with $10 \mathrm{~min}$ ischemia only $5 \mathrm{~min}$ before prolonged ischemic period. As to the duration of injurious ischemia, marked changes in organs like stomach, pancreas or liver are acchieved after 30 to 120 $\min (1,3,9,11)$. Even though our protocol met all the reported criteria for the induction of ischemia and ISP in stomach, CAO was found to lack the ability to produce (and thus also modify) significant liver or pancreatic damage demonstrated by others $(9,5,11,3)$.

\section{Conclusions}

This pilot study demonstrates a new alternative of gastrointestinal interstitial blood perfusion measurement by LM. Under given experimental conditions, the technique 
allowed a detection of selective microcirculation modulation in rat stomach, but failed to detect reported protective potency of local ISP in this organ. Lack of other splanchnic or systemic effects as indicated by absence of change in LM and selected serum biochemical parameters requires further studies.

\section{Acknowledgements}

The authors acknowledge an excellent cooperation with Assoc. prof. Živná, Ms. Ježková and Ms. Kriesfalusyová during the experimental work in the vivarium. We also thank Ms. Malá and Ms. Prokopová for skillful technical assistance with the biochemical analyses.

This work was principally supported by a Research Project from the Czech Ministry of Foreign Commerce (MZO 00179906), and in part also by a grant from the Czech Ministry of Education (MŠMT COST B25).

\section{References}

1. Brzozowski T, Konturek PC, Pajdo R, et al. Importance of brain-gut axis in the gastroprotection induced by gastric and remote preconditioning. J Physio Pharmacol 2004;55:165-77.

2. de la Pena A, Liu P, Derendorf H. Microdialysis in peripheral tissues. Adv Drug Deliv Rev 2000;45:189-216.

3. Dembinski A, Warzecha Z, Ceranowicz P, et al. Effect of ischemic preconditioning on pancreatic regeneration and pancreatic expression of vascular endothelial growth factor and platelet-derived growth factor-A in ischemia/ reperfusion-induced pancreatitis. J Physiol Pharmacol 2006;57:39-58.
4. Gho BC, Schoemaker RG, van den Doel MA, Duncker DJ, Verdouw PD. Myocardial protection by brief ischemia in noncardiac tissue. Circulation 1996;94:2193-200

5. Gong JP, Tu B, Wang W, Peng Y, Li SB, Yan LN. Protective effect of nitric oxide induced by ischemic preconditioning on reperfusion injury of rat liver graft. World J Gastroenterol 2004;10:73-6.

6. Hotter G, Closa D, Prados M, et al. Intestinal preconditioning is mediated by a transient increase in nitric oxide. Biochem Biophys Res Commun 1996; 222:27-32.

7. Hrubá $\mathrm{P}$, Živný $\mathrm{P}$, Živná H, Palička V. Muscle, liver and kidney interstitium blood flow changes in rats measured by microdialysis with flow marker added. Klin Biochem Metab 2004;12:9-13.

8. Jedličková V, Paluch Z, Alusik S. Determination of nitrate and nitrite by high-performance liquid chromatography in human plasma. J Chromatogr B Analyt Technol Biomed Life Sci 2002;780:193-7.

9. Koti RS, Yang W, Dashwood MR, Davidson BR, Seifalian AM. Effect of ischemic preconditioning on hepatic microcirculation and function in a rat model of ischemia reperfusion injury. Liver Transpl 2002;8:1182-91.

10. Mallick IH, Yang W, Winslet MC, Seifalian AM. Ischaemic preconditioning improves microvascular perfusion and oxygenation following reperfusion injury of the intestine. Br J Surg 2005;92:1169-76.

11. Obermaier R, von Dobschuetz E, Drognitz O, Hopt UT, Benz S. Ischemic preconditioning attenuates capillary no-reflow and leukocyte adherence in postischemic pancreatitis. Langenbecks Arch Surg 2004;389:511-6.

12. Pajdo R, Brzozowski T, Konturek PC, et al. Ischemic preconditioning, the most effective gastroprotective intervention: involvement of prostaglandins, nitric oxide, adenosine and sensory nerves. Eur J Pharmacol 2001;427:263-76.

13. Peralta C, Closa D, Xaus C, Gelpi E, Rosello-Catafau J, Hotter G. Hepatic preconditioning in rats is defined by a balance of adenosine and xanthine. Hepatology 1998;28:768-73

14. Peralta C, Serafin A, Fernandez-Zabalegui L, Wu ZY, Rosello-Catafau J. Liver ischemic preconditioning: a new strategy for the prevention of ischemia-reperfusion injury. Transplant Proc 2003;35:1800-2.

15. Stallknecht B, Donsmark M, Enevoldsen LH, Fluckey JD, Galbo H. Estimation of rat muscle blood flow by microdialysis probes perfused with ethanol, [14C]ethanol, and 3H2O. J Appl Physiol 1999;86:1054-61.

Submitted August 2006. Accepted September 2006.

\section{Corresponding author:}

Norbert Cibiček, MD, University Hospital Hradec Králové, Institute for Clinical Biochemistry and Diagnostics, Sokolská 581, 50005 Hradec Králové, Czech Republic, e-mail: cibicekn@lfhk.cuni.cz 